\title{
Study on cooling fluid under the condition of the existence of outside force
}

\author{
Kanru Cheng ${ }^{1, a}$ \\ ${ }^{1}$ School of North China Electric Power University (Baoding), Baoding 071000, China \\ a15176265865@163.com
}

\section{Keywords: Newton's law of cooling, natural-cooling, outside-power fluid cooling}

\begin{abstract}
This paper will explain the theory of building a differential equation model that helps the simple water containment vessel offering a constant-temperature. The simple water containment vessel has an open system without the temperature control unit, and the effect of the constant-temperature may be impacted by two main factors: continuous injection of hot water and manpower. Based on all those conditions that mentioned above, this paper will take two steps to work out the appropriate strategy that could resolve this issue: firstly, build a cooling model that could offer a high imitation of the cooling process while user taking the bath; secondly, build a mixed model of hot water and cold water, and then calculate the minimum flow rate that should be able to let the water temperature infinite approaching the initial temperature with the minimum amount of water. The following text will state the whole strategy and program detailed by four main sections: restatement of the problem, basic assumptions and justification, notations and symbol descriptions, and the outside-power fluid cooling model.
\end{abstract}

\section{Introduction}

The sap-style tub is able to offering a constant-temperature bath because of the secondary heating system and circulating jets while a simple water containment vessel could not offering the same service. This paper will explain the theory of building a mathematical model that helps the simple water containment vessel offering a constant-temperature bath. A constant trickle of hot water need to be added from the faucet to reheat the bathing water even throughout the whole bath time. The strategy should be able to let the water temperature infinite approaching the initial temperature with the minimum amount of water, and also realizable. Based on those ideas that mentioned above, it is supposed to consider the sensitivity from the three following factors: the shape and volume of the tub; the shape, volume and temperature of the person in the bathtub; the motions made by the user in the bathtub.

\section{Basic Assumptions and Justifications}

In order to simplify the problem, this paper outlined the following basic assumptions, each of which is properly justified.

1. The water adds into the bathtub won't change from the beginning to the end. Regard the temperature of the water in the hot-water heater hasn't change during the whole process of bathing, therefore, the temperature of the water that add into the bathtub is the same.

2. Water temperature will not decrease when add the water. Regard the heat exchange may not happen during the process of add water.

3. Ignore the minor response of the body temperature in the process of taking a bath. When the water temperature above people's body temperature, the body temperature will have a slightly increase. On the contrary, when the water temperature lower than people's body temperature, the body temperature will has a corresponding decrease. But it could be ignored, because of the slightly change.

4. Considering the constant room temperature. Assuming that the room temperature may not have an obvious change result from the whole process of bathing may not take too much time.

5. Considering the water lose heat only by Heat Convection with bathtub wall, the Thermal Transmission between water and the air, the Thermal Transmission between water and people's body. Since the model will be installed inside the room, the main reason that the water in the bathtub stop heating should be the Heat Convection. Thus, the losing of the radiation heat and the evaporative heat might be ignored.

6 . The density and viscosity of the water in the bathtub will not change in the bath process before put in the bubble bath additive. Small changes in the density and viscosity might be ignored. 


\section{Symbol Descriptions}

All the variables and constant used in this paper are listed in Table 1.

Table 1 Symbol Table

\begin{tabular}{lll}
\hline Symbol & Definition & Units \\
\hline $\mathrm{Q}$ & Quantity of heat & $\mathrm{J}$ \\
$\mathrm{h}$ & Convective heat-transfer coefficient & $\mathrm{W} /(\mathrm{m} 2 \cdot \mathrm{K})$ \\
$\mathrm{C}$ & Heat Capacity & $\mathrm{J} / \mathrm{K}$ \\
$\alpha$ & Thermal conductivity of the bathtub & $\mathrm{W} /(\mathrm{m} \cdot \mathrm{K})$ \\
$\mathrm{d}$ & The thickness of the bathtub wall & $\mathrm{m}$ \\
$S w-a$ & Water surface and air contact area & $\mathrm{m} 2$ \\
$S b$ & The surface area of bathtub & $\mathrm{m} 2$ \\
$S h$ & The surface area of people & $\mathrm{m} 2$ \\
$T 0$ & The initial temperature of water & ${ }^{\circ} \mathrm{C}$ \\
$T a$ & The temperature of the air & ${ }^{\circ} \mathrm{C}$ \\
$T l$ & The temperature of the current & ${ }^{\circ} \mathrm{C}$ \\
$T w(t)$ & The temperature of the water in bath tub at time t & ${ }^{\circ} \mathrm{C}$ \\
$\rho$ & The density of water & $\mathrm{kg} / m 3$ \\
\hline
\end{tabular}

\section{The Outside-Power Fluid Cooling Model}

We will start with the idea of the cooling model. This model mainly based on Newton's law of cooling and it explains the water cooling process in three main ways: Heat Convection with bathtub wall, Thermal Transmission between water and the air, the Thermal Transmission between water and people's body.

\section{A The Natural-cooling Model}

Firstly, a model is built to describe the cooling condition of the water in the bathtub when it is in the natural condition without the person's impact Newton's law of cooling is the law that the object follows that transfer heat into the surrounding medium when its temperature is higher than the surroundings, expressed in a formula is that

$$
\mathrm{Q}=S_{w-a} h \Delta t
$$

In formula (1), the $\boldsymbol{S}_{\boldsymbol{w}-\boldsymbol{a}}$ refers to the contact area of water surface and air, $\boldsymbol{h}$ refers to the convective heat-transfer coefficient of water, $\Delta \boldsymbol{t}$ refers to the variation of the water temperature. Exchanging the above formula into the differential equation form, The object (i.e., thermal system) is under the condition of natural cooling, when the experimental system's temperature $T_{w}(t)$ higher than the outer circumstance temperature $T a$, and at the same time, the difference between $T_{w}(t)$ and $T a$ is very small, the heat loss of the system's surface is in proportion to $\left(T_{w}(\mathrm{t})-T_{a}\right)$, that is

$$
\frac{d Q}{d t}=k_{0}\left[T_{W}(t)-T_{a}\right]
$$

The formula (2) is a differential equation about quantity of heat and time. $\boldsymbol{k}_{\mathbf{0}}$ is a coefficient that equals to $\boldsymbol{S}_{\boldsymbol{w}-\boldsymbol{a}}$ multiply $\boldsymbol{h}$, therefore, formula (2) could be write as

$$
\mathrm{dQ}=S_{w-a} h\left[T_{W}(t)-T_{a}\right]
$$

The Heat Capacity of the system is $\boldsymbol{C}$, after losing heat to $\boldsymbol{d Q}$, the water temperature has changed $\boldsymbol{d T}$, thus a equation could be arrived: $\boldsymbol{d} \boldsymbol{Q}=\boldsymbol{C} \boldsymbol{\boldsymbol { d }} \boldsymbol{T}$, enter this relationship into formula(3)

$$
\Delta \mathrm{T}^{\prime}=\frac{S_{w-a} h}{c}\left[T_{W}(t)-T_{a}\right]
$$

According to formula (4), figure out the integral equation of $\Delta \boldsymbol{T}^{\prime}$ the change of temperature that caused by Thermal Transmission

$$
\Delta \mathrm{T}^{\prime}=\frac{S_{w-a} h}{c} \int_{t_{0}}^{t}\left[T_{W}(t)-T_{a}\right] d t
$$

In the same way, the integral equation about Heat Convection between the water and bathtub could be arrived

$$
\Delta \mathrm{T}^{\prime \prime}=\frac{S_{b} \alpha}{C \cdot d} \int_{t_{0}}^{t}\left[T_{W}(t)-T_{a}\right] d t
$$


By the knowledge of thermodynamics known, considering the ratio of the heat loss through Thermal Transmission and the heat loss through Heat Convection in the bathtub is equal to the ratio of the water surface and air contact area and the surface area of bathtub, then the equation could be arrived

$$
\Delta \mathrm{T}=\frac{9}{10} \cdot \frac{S_{w-a} h}{C} \int_{t_{0}}^{t}\left[T_{W}(t)-T_{a}\right] d t+\frac{1}{10} \cdot \frac{S_{b} \alpha}{C \cdot d} \int_{t_{0}}^{t}\left[T_{W}(t)-T_{a}\right] d t
$$

Calculate formula (7) the derivation of variable upper limit equation and get

$$
\frac{d T_{w}(t)}{d t}=\left[\frac{9 S_{w-a h}}{10 C}+\frac{S_{b} \alpha}{10 C d}\right] \cdot\left[T_{W}(t)-T_{a}\right]
$$

One thing that need to be highlighted is valuing the parameter $\boldsymbol{h} . \boldsymbol{h}$ is the Convective heat-transfer coefficient of the water, the evaluation of it depends on many factors include the flow velocity of water $\boldsymbol{u}$, the density of water $\boldsymbol{\rho}$, the viscosity of water $\boldsymbol{\mu}$, the heat conduction coefficient of water $\boldsymbol{k}$ and the buoyancy caused by temperature difference $\boldsymbol{\Delta t}$ express as $\boldsymbol{\beta} \boldsymbol{g} \boldsymbol{\Delta} \boldsymbol{t}$. Then get the function of $\boldsymbol{h}$

$$
h=f(u, l, \mu, \rho, k, \beta g \Delta t)
$$

In formula (9), by using Actor Analysis Method could get three empirical correlation equations: $N u=\frac{h l}{k}$, $R e=\frac{l u \rho}{\mu}, G r=\frac{l^{3} \rho^{2} \beta g \Delta t}{\mu^{2}}$. These three equations reflects the relationship between these variables. Because the function of $\boldsymbol{h}$ cannot get an exact expression, the general method could not be used in getting the expression of $T_{w}(t)$ by formula(8).In order to get the final result of $T_{w}(t)$, there are three steps need to be take: Firstly, using the Classical fourth-order Runge-Kutta Method to get this equation's numerical solution .Select 240 points in the range of 0 to 1200 seconds homogeneously, and calculate the corresponding result, then draw up the picture of the point. Secondly using the least square method get a fit curve, and make out the formula of the fit curve.

\section{B Analysis on the Specific Case}

To study the model's rationality, choose a specific case of the model. First, making some assumptions, the specific data are shown in Table 2.

Table 2

\begin{tabular}{ll}
\hline Factor & Date \\
\hline Bathtub length 1 & $1.5 \mathrm{~m}$ \\
Bathtub width & $0.8 \mathrm{~m}$ \\
Bathtub tall & $0.5 \mathrm{~m}$ \\
The density of water & $103 \mathrm{~kg} / \mathrm{m} 3$ \\
The mass of water & $0.6 \mathrm{~kg}$ \\
Heat Capacity & $2502 \mathrm{~J} / \mathrm{K}$ \\
h & $200 \mathrm{~W} /(\mathrm{m} 2 \cdot \mathrm{K})$ \\
aacrylic2 & $19 \mathrm{~W} /(\mathrm{m} \cdot \mathrm{K})$ \\
aceramic & $319 \mathrm{~W} /(\mathrm{m} \cdot \mathrm{K})$ \\
\hline
\end{tabular}

Note: 1 Choose the square bath tubas the research object.2Different material has different thermal conductivity $\alpha$. The material of bathtub is usually be ceramic or acrylic, $\boldsymbol{\alpha}_{\text {ceramic }}$ is $319 \mathrm{~W} /(\mathrm{m} \cdot \mathrm{K})$ and $\boldsymbol{\alpha}$ acrylic is $19 \mathrm{~W} /(\mathrm{m} \cdot \mathrm{K})$.

Fig.1 describes two different scatter diagram under two different $\boldsymbol{\alpha}$. Red points represent ceramic's change while green points represent acrylic's change. 


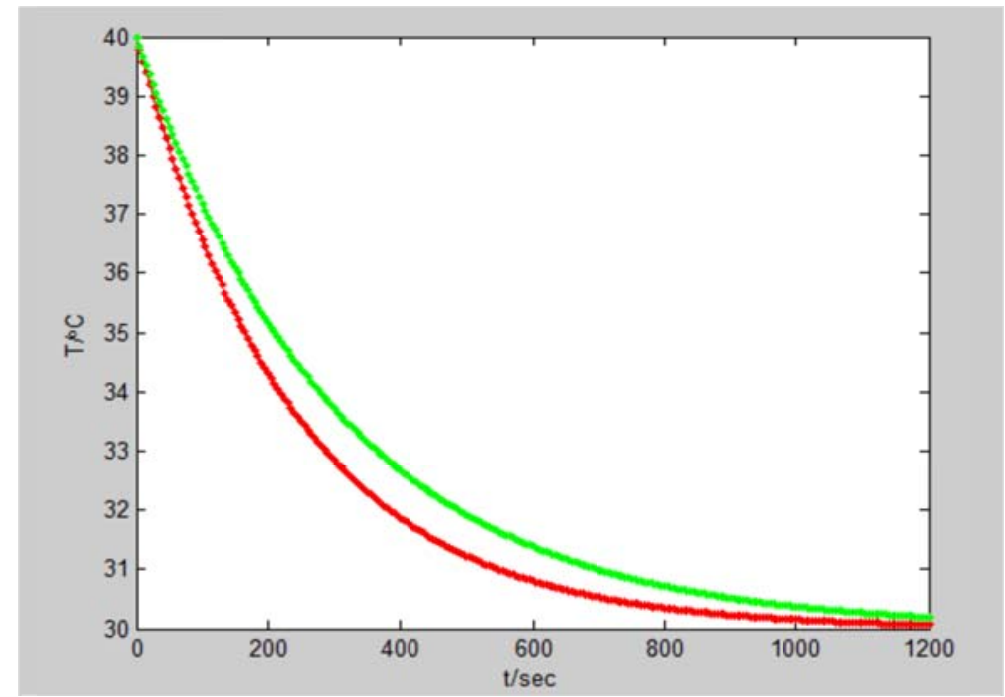

Fig.1 Time -temperature scatter diagram

Fig.1 reflect the approximate trend of the cooling process, and using the least square method to get a fit curve, for instance, in Fig.2, the black curve is the fit curve. Here only make the fit curve of the acrylic.

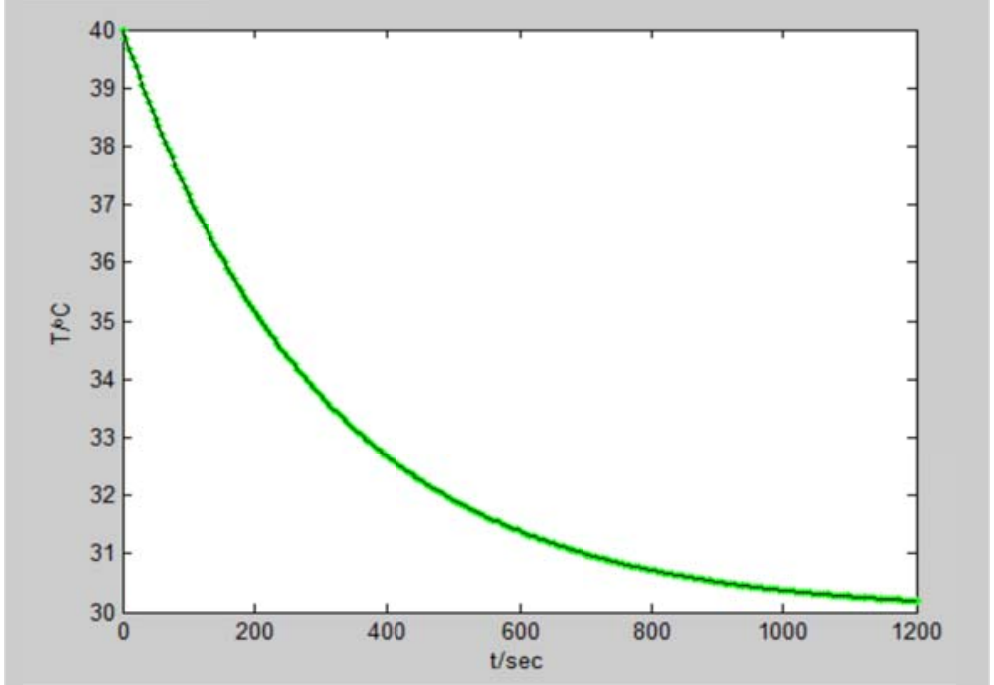

Fig.2 Time -temperature scatter diagram and fit diagram

It is not very hard to see that the distribution of the scatter diagram and the fit curve is highly coincident, then make out the formula of the curve, that is

$$
T_{w}(t)=10 e^{-0.0033 t}+30
$$

This formula reflect the cooling process under a specific case.

\section{The Outside-Power Fluid Cooling Model}

The user's impact to the water seems to be a significant factor in the cooling process among the whole bath process. A Outside-Power Fluid Cooling Model needs to be built with taking the person's shape, volume and motions into the Natural-cooling Model. First of all, analyzing the user's impact to the water in the cooling process. Here adopt the analogy analysis method, the user's limbs and body could be simplified into cylindrical solid, so the process of Thermal Transmission between water and the user's body could be regarded as the heat transfer between fluid and the tube when fluid flows across the circular tube, the basic principle diagram as shown in Fig.3. 


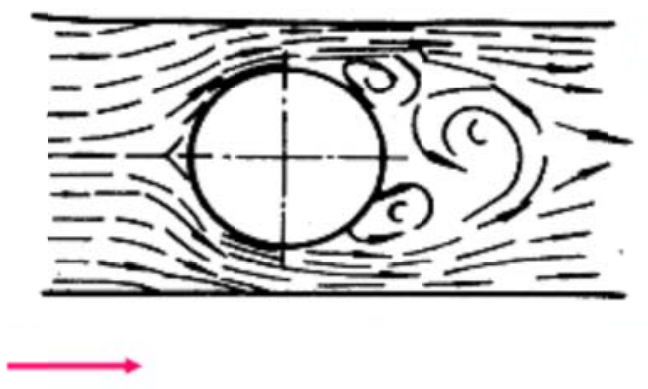

Fig.3 People in the water schematic diagram

In Fig.3, it is easy to get a curve of the convective heat-transfer coefficient $h$ related to the angle near the user by comparing the human body to circular tube, as shown in Fig.4.

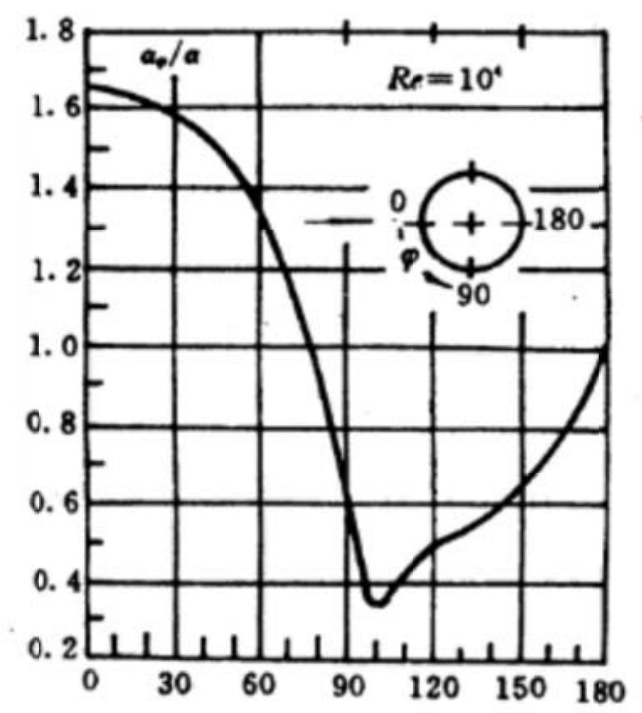

Fig.4 Convective heat-transfer coefficient curve

Then, a integral equation of the change of temperature about the Thermal Transmission between water and the user could be make out, that is

$$
\Delta \mathrm{T}^{\prime \prime \prime}=\frac{S_{h} h}{C} \int_{t_{0}}^{t}\left[T_{W}(t)-T_{a}\right] d t
$$

Take the user's impact into the Natural-cooling Model, and combining formula (10) and formula (11), then making some deformation could get the final formula

$$
\Delta \mathrm{T}=\frac{9}{10} \cdot \frac{S_{w-a} h}{C} \int_{t_{0}}^{t}\left[T_{W}(t)-T_{a}\right] d t+\frac{1}{10}\left[\frac{S_{b}^{2} \alpha}{\left(S_{b}+S_{h}\right) C d} \int_{t_{0}}^{t}\left[T_{W}(t)-T_{a}\right] d t+\frac{S_{b}^{2} h}{\left(S_{b}+S_{h}\right) C} \int_{t_{0}}^{t}\left[T_{W}(t)-T_{a}\right] d t\right.
$$

In the same way, valuing the parameter $\boldsymbol{h}$ also need to be highlighted. $\boldsymbol{h}$ is the Convective heat-transfer coefficient of the water, when the person in this cooling system, the evaluation of it depends on five factors: the flow velocity of water $\boldsymbol{u}$, the density of water $\boldsymbol{\rho}$, the viscosity of water $\boldsymbol{\mu}$, the heat conduction coefficient of water $\boldsymbol{k}$ and the heat capacity of water $\boldsymbol{C} \boldsymbol{p}$. Then get the function of $\boldsymbol{h}$

$$
h=f\left(u, l, \mu, \rho, k, C_{p}\right)
$$

In formula (13), by using the Actor Analysis Method could get three empirical correlation equations: $N u=\frac{h l}{k}, \operatorname{Re}=\frac{l u \rho}{\mu}, \operatorname{Pr}=\frac{C_{p} \mu}{k}$. These three equations reflects the relationship between these variables. Because the function of $\boldsymbol{h}$ cannot get an exact expression, the general methods could not be used in getting the expression of $T_{w}(t)$ by formula(12).In order to get the final result of $T_{w}(t)$, the first step is using the Classical fourth-order Runge-Kutta Method to get this equation's numerical solution. Select 240 points in the range of 0 to 1200 seconds homogeneous, suppose the room temperature is $30^{\circ} \mathrm{C}$, and calculate the corresponding result, then draw up the picture of the point, get the scatter diagram. Secondly, using the least square method to get a fit curve and get the formula of the curve. 


\section{Applying the improved model to a specific Case}

Use the data in Table 2 get the scatter diagram of acrylic as Fig.5.

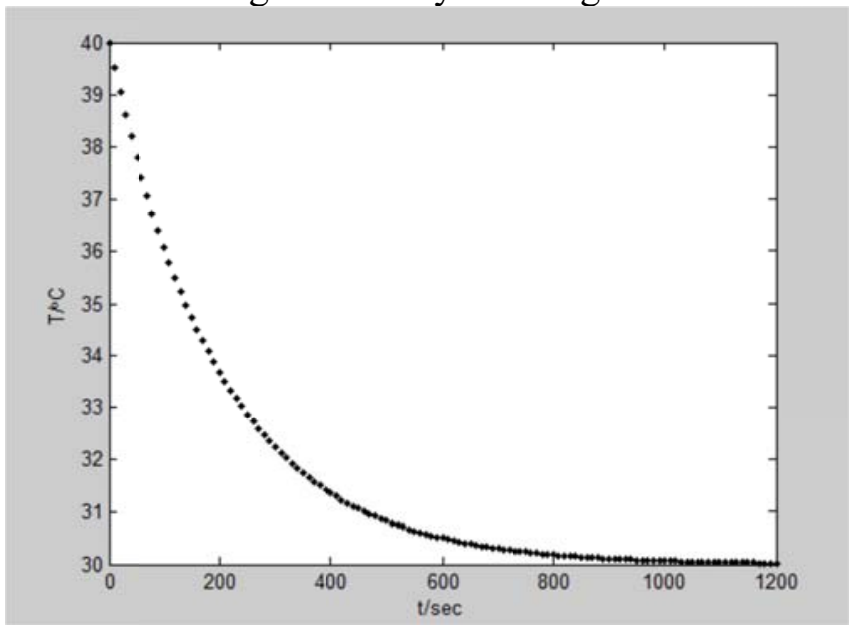

Fig.5 Time -temperature scatter diagram

Fig.5 reflect the approximate trend of the cooling process, and using the least square method to get a fit curve, as the Fig.6 shown

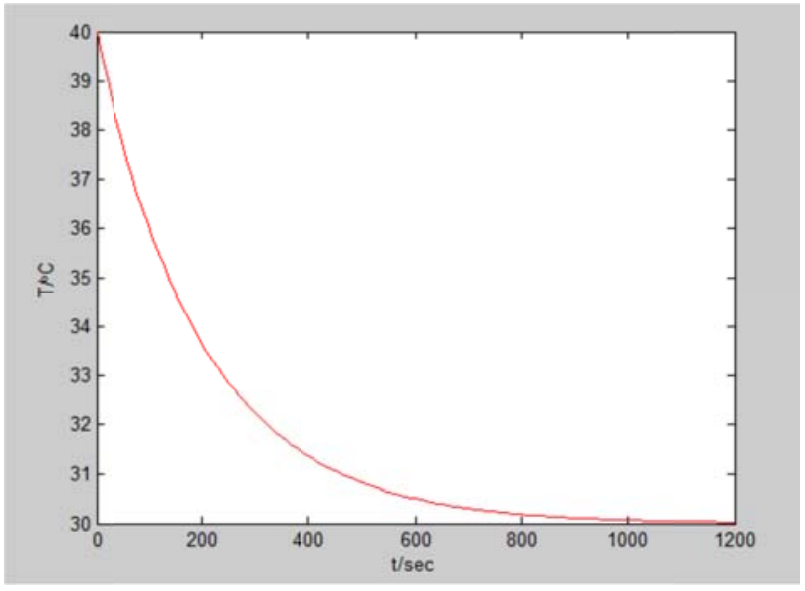

Fig.6 Fit diagram of acrylic's cooling process scatter diagram

Then calculate the formula of the curve, that is

$$
T_{w}(t)=10 e^{-0.005 t}+30
$$

\section{References}

[1] Winterton, R.H.S. Newton's law of cooling .Contemporary Physics, v 40, n 3,205-12, May-June 1999.

[2]Liu Zhihua, Liu Ruijin. Study on Cooling Regularity for Newton's Law of Cooling. Journal of Shandong University of Technology(Sci\&Tech).

[3] Ye Lijuan. Convection Heat Transfer Coefficient of Numeral Connection Type. Journal of Chenzhou Teachers College.

[4]Heinisch, Rudolf; Rogowski, Andreas; Schuett, Ehrenfried. First law of thermodynamics. Forschung im Ingenieurwesen/Engineering Research, v 60, n 1-2, p 29-35, Jan-Feb 1994.

[5]Lu, Xiaoping; Yu, Shurong; Yu, Jianping; He, Ailing; Guo, Dandan . Energy transfer conversion mechanism and minimum entropy generation principle for convective heat transfer enhancement. Chemical Industry Press.

[6Jiang Zhengrong; Zhu Guoliang. Concise Construction Manual Calculation. China Building Industry Press.

[7]Kamei, Shuya; Isshiki, Seita; Takahashi, Akira1; Azima, Toshio. Visualization study of the stream line and secondary flow in the running water bathtub.Proceedings of SPIE - The International Society for Optical Engineering, v 4948, p 823-830, 2002 